Received: 2018.05 .23

Accepted: 2018.07.31

Published: 2018.09 .05

\title{
Distinct Anti-Fibrotic Effects of Exosomes Derived from Endothelial Colony-Forming Cells Cultured Under Normoxia and Hypoxia
}

Authors' Contribution: Study Design A Data Collection B Statistical Analysis C Data Interpretation D Manuscript Preparation E Literature Search F Funds Collection G
BCDEF 1,2 WenHao Liu*

CE 1,2 HaiFeng Zhang*

AF 1,2 JingTing Mai*

BDF 1,2 ZhiTeng Chen

DF 1,2 TuCheng Huang

B 1,2 ShaoHua Wang

AE 1,2 YangXin Chen

AE 1,2 JingFeng Wang
1 Department of Cardiology, Sun Yat-sen Memorial Hospital, Sun Yat-sen University, Guangzhou, Guangdong, P.R. China

2 Guangdong Province Key Laboratory of Arrhythmia and Electrophysiology, Guangzhou, Guangdong, P.R. China
Corresponding Authors: Source of support:

Material/Methods:

Results:

Conclusions:

MeSH Keywords:

Full-text PDF:
* These authors contributed equally to this work and should be considered co-first authors YangXin Chen, e-mail: tjcyx1995@163.com, JingFeng Wang, e-mail: dr_wjf@vip.163.com This work was supported by grants from the National Natural Science Foundation (grants no. 81570213, 81570329, 81500307, 81700215, and 81300071), the Natural Science Foundation of Guangdong Province of China (grants no. $2015 \mathrm{~B} 010131010$ and 2016A030313263), and the Science and Technology Program of Guangzhou (grants no. 2014Y2-00118 and 201510010048)

Background: The therapeutic potential of endothelial colony-forming cells (ECFCs) may be impaired in an ischemic environment. Direct injection of ECFCs is not an effective method of rescuing the ischemic heart, but exosomes derived from these cells may be a promising therapeutic tool. However, exosomes produced under normoxia and hypoxia may not be identical. Therefore, the purpose of this study was to investigate alterations in the anti-fibrotic effects of hypoxia-treated ECFC-derived exosomes and the underlying mechanism involved.

ECFCs were isolated from peripheral blood and exosomes were collected from ECFCs treated with normoxia (nor-exo) or hypoxia (hyp-exo). Effects of exosomes on cardiac fibroblast activation were evaluated in vitro. MicroRNAs (miRNAs) inside the exosomes were extracted and compared using next-generation RNA sequencing. Predicted target mRNAs of miR-10b-5p were validated using a dual-luciferase reporter gene assay method. Nor-exo significantly ameliorated cardiac fibroblast activation in vitro. These effects were attenuated in the hypexo-treated group. miR-10b-5p was enriched in nor-exo but not in hyp-exo. Dual-luciferase reporter gene assay found that both SMAD-specific E3 ubiquitin protein ligase 1 (Smurf1) and histone deacetylase 4 (HDAC4) mRNAs were inhibited by miR-10b-5p. The expression of neutral sphingomyelinase 2 (N-SMase2) was decreased in hypoxia ECFCs, and this result was consistent with the changes in miR-10b-5p in hyp-exo.

Due to a reduction of miR-10b-5p, which targets the fibrotic genes Smurf1 and HDAC4, the anti-fibrotic effects of hyp-exo were abolished.

Adult Stem Cells • Cell Hypoxia • Exosomes • Fibroblasts • MicroRNAs

https://www.medscimonit.com/abstract/index/idArt/911306

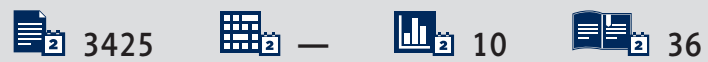




\section{Background}

Cardiovascular disease is one of the leading causes of morbidity and mortality worldwide. Acute myocardial infarction (AMI) is a major contributor to poor outcomes, particularly in developed countries [1,2]. Cardiac fibrosis, which can occur secondary to AMI, has drawn increasing attention because it induces a wide range of chronic adverse events (e.g., heart failure and arrhythmia).

During the past decade, cell-based therapies have grown to be one of the most promising methods to treat cardiac fibrosis. In particular, the injection of stem cells derived from various sources has been a focus of scientific interest [3,4]. Several studies have reported that endothelial colony-forming cells (ECFCs), known as progenitor cells of endothelial cells, reduce fibrosis in the post-infarct heart due to their high proliferative potential [4-6]. In addition to the direct effects of ECFCs, an increasing number of researchers have reported that extracellular vesicles derived from ECFCs, specifically exosomes, play a key role in mediating therapeutic effects against fibrosis $[7,8]$. Exosomes are small membrane-bound vesicles that have a diameter of approximately $30-150 \mathrm{~nm}$. They are present in all body fluids and have recently received attention because of their potential role as communication vehicles between cells [9].

The direct usage of ECFCs is associated with certain problems. Importantly, the extracellular environment required to support ECFC growth and function is destroyed due to cardiac ischemia $[10,11]$. For example, previous studies have shown that less than $10 \%$ of injected stem cells are detected in the infarcted myocardium $[12,13]$. Furthermore, several studies have found that the proliferation, migration, and tube-formation abilities of ECFCs are decreased under long-term hypoxic conditions [14-16]. On the contrast, use of ECFC-derived exosomes is quite promising. Burger first reported that ECFCs protect against acute kidney injury by exosomes [17]. Vinas et al. subsequently reported that ECFC-derived exosomes reduce ischemic kidney injury by transferring miRNAs [7].

Because of the research progress made in use of exosomes, many believe that ECFCs can benefit ischemic organs, as in cardiac ischemia. However, it remains unclear whether the therapeutic effects of ECFC-derived exosomes are mitigated under ischemic conditions. Therefore, determining the effects of ECFC-derived exosomes produced under ischemic conditions is very important. Moreover, if ischemia/hypoxia attenuates the effects of ECFC-derived exosomes, it may support use of exosomes therapy in clinical practice instead of ECFCs therapy. Since hypoxia is one of the most important pathophysiologic changes in the ischemic heart, we examined the responses of ECFC-derived exosome fractions to low oxygen in vitro.
In this study, we focused on the anti-fibrotic effects of ECFCderived exosomes.

\section{Material and Methods}

\section{ECFC isolation and characterization}

ECFCs were isolated, cultured, and characterized as we previously described (see Supplementary Materials and Supplementary Figure 1) [18]. The peripheral blood used in the experiment was donated by healthy volunteers. All volunteers signed informed consent and this program was approved by the Clinical Ethics Committee of Sun Yat-sen Memorial Hospital.

\section{Hypoxia treatment, exosome isolation and analysis}

Prior to exosome isolation, ECFCs were cultured in EGM-2 medium with $2 \%$ serum replacement (15950-017, PALL Life Science, USA) $[19,20]$ to avoid exosomes from fetal bovine serum [21] and then were divided into normoxia and hypoxia groups. A humidified, temperature-controlled hypoxia chamber (Galaxy ${ }^{\oplus} 48$ R incubator, Eppendorf/Galaxy Corporation, USA) was used to maintain cells in $1 \% \mathrm{O}_{2}$. We collected conditioned media after $72 \mathrm{~h}$.

Conditioned media were subjected to sequential centrifugation (Optima L 100XP ultracentrifuge, Beckman Coulter SW 41 Ti rotor, USA) at $10000 \mathrm{rpm}$ for $30 \mathrm{~min}$ to remove microparticles and $100000 \mathrm{rpm}$ for $70 \mathrm{~min}$ followed by washing once with PBS $[22,23]$. Pellets were resuspended with PBS and then filtered using a $0.22-\mu \mathrm{m}$ sterile filter. Next, the samples were centrifuged at $100000 \mathrm{rpm}$ for $70 \mathrm{~min}$. Normoxia-treated ECFCderived exosomes (nor-exo) and hypoxia-treated ECFC-derived exosomes (hyp-exo) were obtained. The exosome pellets were suspended, and the protein contents of the pellets were analyzed using a Micro BCA Protein Assay Kit (P0012S, Beyotime, China).

The ultrastructures of the exosomes were detected by transmission electron microscopy (TEM) (JEOL JEM-1400, Japan) [24]. Exosome sizing was performed via nanoparticle tracking analysis with a NanoSight LM10 instrument (NanoSight, Ltd., Malvern, UK) [23,24]. Protein markers, such as CD63, CD9, Alix and calnexin, were examined by immunoblotting [23]. In brief, exosomes were resolved by sodium dodecyl sulfate-polyacrylamide gel electrophoresis (SDS-PAGE) and then transferred onto polyvinylidene difluoride (PVDF) membranes (IPVH00010, Millipore, USA) before incubating with primary antibodies (CD9, catalog no. ab92726, UK; CD81, catalog no. ab109201, UK; Alix, catalog no. ab186728, UK; CD63, catalog no. sc-15363). After washing with TBST, membranes were incubated with anti-lgG horseradish peroxidase-conjugated secondary antibody (anti-rabbit lgG, catalog (ST no. 7074, USA) for $60 \mathrm{~min}$ at room temperature. 
After extensive washing, bands were detected by enhanced chemiluminescence. Band intensities were quantified using imaging software (ImageJ, version 1.50i).

\section{Cardiac fibroblast cultivation and activation}

Human cardiac fibroblasts from adult ventricles were purchased from ScienCell Research Laboratories (catalog no. 6310, lot no. 9617, USA). Cells were cultured in Fibroblast Medium-2 (FM-2, catalog no. 2331, ScienCell, USA), and cells at passage 5 were used in a fibroblast activation assay. After the initiation of TGF- $\beta$ (catalog no. 100-21, PeproTech, USA) stimulation $(15 \mathrm{ng} / \mathrm{ml})$, cardiac fibroblasts were cultured in basal medium with $0.1 \%$ fetal bovine serum for $48 \mathrm{~h}$. Next, $75 \mu \mathrm{g} / \mathrm{ml}$ nor-exo or hyp-exo were added, and the effects of the exosomes were tested in vitro (see Supplemental Figure 2 for dose testing).

\section{Western blotting analysis}

Protein levels were measured by Western blot analysis as previously reported [25]. Cells were washed 3 times with PBS before collection and lysed with modified RIPA buffer. Cells were completely lysed after repeated vortexing, and supernatants were acquired through centrifugation at $14000 \mathrm{rpm}$ for 30 min. Proteins were resolved by sodium dodecyl sulfate-polyacrylamide gel electrophoresis (SDS-PAGE) and then transferred onto polyvinylidene difluoride (PVDF) membranes (IPVH00010, Millipore, USA) before incubating with primary antibodies ( $\alpha$ smooth muscle actin ( $\alpha$-SMA), catalog no. ab5694, UK; collagen I (Col-1 $\alpha$ ), catalog no. ab138492, UK; and GAPDH, catalog CST no. 2118, USA). After washing with TBST, membranes were incubated with anti-lgG horseradish peroxidase-conjugated secondary antibody (anti-rabbit IgG, catalog CST no. 7074, USA) for $60 \mathrm{~min}$ at room temperature. After extensive washing, bands were detected by enhanced chemiluminescence. Band intensities were quantified using imaging software (ImageJ, version 1.50i).

\section{Immunocytochemistry}

Cells were fixed in 4\% (w/v) paraformaldehyde for $20 \mathrm{~min}$ at room temperature. Following PBS washes, cells were incubated with $0.1 \%$ Triton X-100 for 10 min at RT and then blocked in $1 \%$ bovine serum albumin (BSA) for $2 \mathrm{~h}$ in PBS before overnight incubation in primary antibody at $4^{\circ} \mathrm{C}$. After washing with PBS, the cells were incubated with the appropriate secondary antibody for $1 \mathrm{~h}$ at RT and imaged by a fluorescence microscope (Leica, German) equipped with a CCD camera (Tokyo, Japan). Primary antibodies, such as anti-collagen I (catalog no. ab138492, UK), were used to stain Col- $1 \alpha$ in fibroblasts. Goat anti-rabbit Alexa Fluor488 IgG (catalog no. ab150077, UK) was used as the secondary antibody. Mounting medium contained the nuclear stain DAPI (D1306, Invitrogen, USA).

\section{Next-generation sequencing and bioinformatics}

Template libraries were prepared from total RNA obtained from the ECFC exosomes. Two independent libraries were generated from nor-exo and hyp-exo, respectively. Each group consisted of 3 independent cultures. RNA segments of different sizes were separated by PAGE, and an 18- to 30-nt band was selected and recycled. Adapters specifically targeting miRNAs and other small RNAs were ligated to each end of the RNA molecule, and an RT reaction was performed to create single-stranded cDNA. Next, adapter-ligated fragments were amplified by PCR. We recovered the purified PCR construct by PAGE, dissolved the recycled products in EB solution, and completed library construction. Data cleaning analysis was performed on the 49-nt sequence tags obtained from HiSeq sequencing, and standard analysis annotated the clean tags into different categories. After obtaining the miRNA results, miRNA target prediction and KEGG pathway analysis for target genes were performed.

\section{miRNA isolation and real-time PCR}

Total RNA was isolated from exosomes using a SeraMirm Exosome RNA Amplification Kit (Catalog RA800A-1, SBI, USA) [26]. A PrimeScript ${ }^{T M}$ RT Reagent Kit (RR037A, TAKARA, Japan) was used for CDNA preparation. Real-time PCR was performed in a Roche LightCycler ${ }^{\circledR} 480$ Real-Time PCR System (Roche, Switzerland). Mamm-U6 RNA was used to normalize differences in RNA levels in each sample. The relative amount of miRNA to U6 RNA was expressed using the $2^{-\Delta \Delta C t}$ method.

The primers were: hsa-miR-10b-5p, reverse transcriptase sequence 5'-GTCGTATCCAGTGCAGGGTCCGAG GTATTCGCACTGGATACGACCACAAA-3', forward sequence 5'-GCGCGGTACCCTGTAGAA-3', reverse sequence 5'-CCA GTGCAGGGTCCG AGGTA-3'. has-U6, reverse transcriptase sequence 5'-AAAATATGGAACGCTTCACG-3', forward sequence 5'-CGCTTCGGCAGCACATATACTA-3', reverse sequence 5'-GCGAGC ACAGAATTAATACGAC-3'.

\section{Dual-luciferase reporter gene assay}

The 3'UTRs of histone deacetylase 4 (HDAC4), inhibitor of DNA binding 2 (ID2), neuroblastoma 1 (NBL1), and SMAD-specific E3 ubiquitin protein ligase 1 (SMURF1) were cloned into the pMIR-REPORT vector immediately downstream of the stop codon of the luciferase gene using standard procedures to generate the HDAC4-3'UTR, ID2-3'UTR, NBL1-3'UTR, and SMURF13'UTR luciferase reporter plasmids, respectively.

HEK293T cells were seeded onto 12-well plates the day before transfection and then transfected with either miR-10b$5 p$ mimics or miR-NC using Lipofectamine ${ }^{\circledR}$ RNAiMAX Reagent 


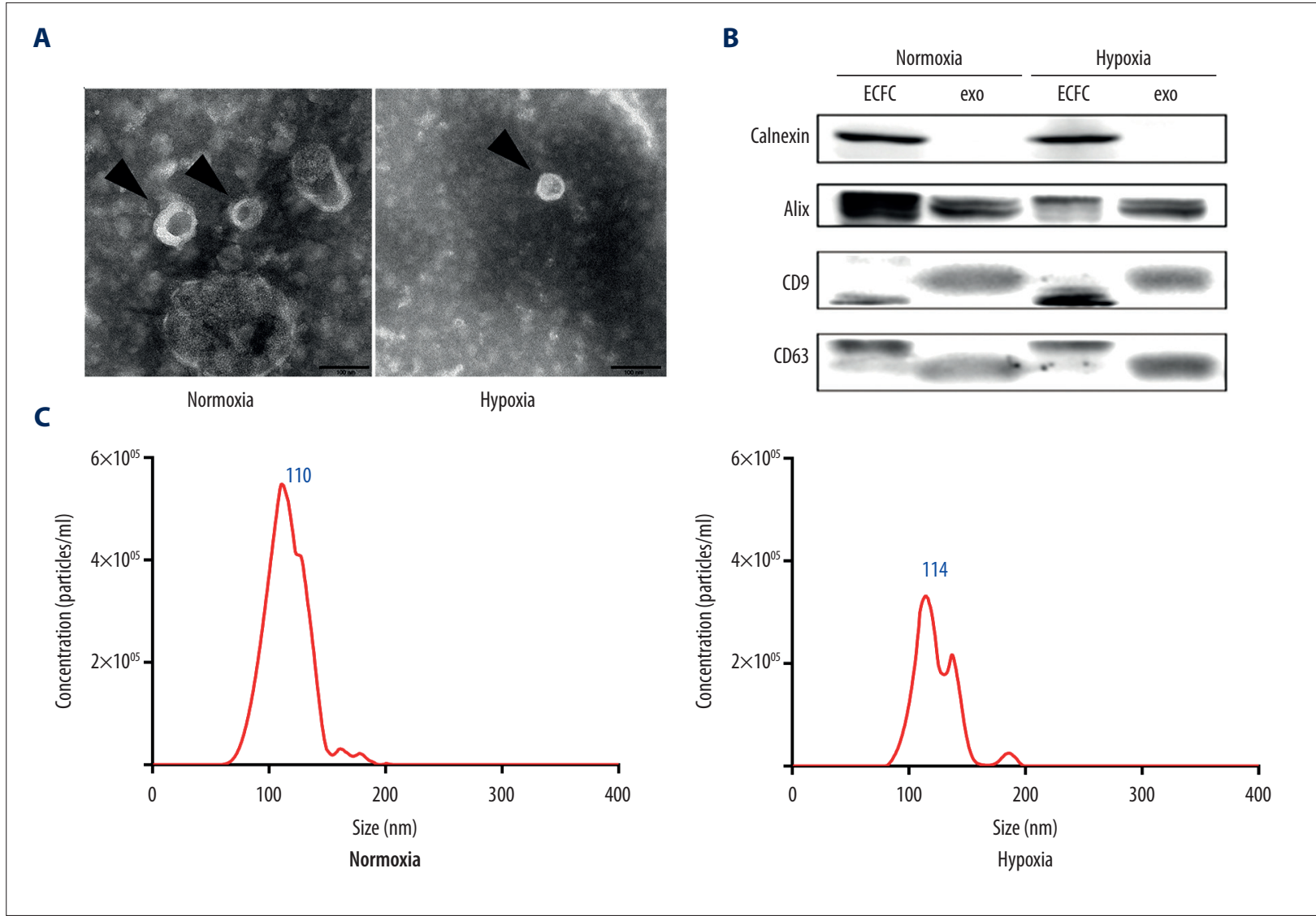

Figure 1. Characteristics of exosomes. (A) Arrowheads indicate ultrastructures of nor-exo and hyp-exo using TEM, bar size $100 \mathrm{~nm}$. (B) CD63, CD9, Alix, and calnexin expression in exosomes. A total of $20 \mu \mathrm{g}$ of protein from lysed ECFCs and $20 \mathrm{mg}$ of protein from lysed exosomes was loaded into each lane (representative image, $n=3$ ). (C) Size distribution profiles of nor-exo and hyp-exo by NanoSight tracking analysis.

(Thermo Fisher Scientific). After $24 \mathrm{~h}$, the cells were transfected with either HDAC4-3'UTR, ID2-3'UTR, NBL1-3'UTR, SMURF13'UTR, or PMIR-REPORT vector together with a Renilla luciferase-expressing vector using Lipofectamine ${ }^{\circledR} 3000$ Reagent (Thermo Fisher Scientific, USA). After 36 h, cells were harvested, and luciferase activities were detected using a DualLuciferase Reporter System (Promega, USA). Three independent experiments were performed, and the calculated means and standard deviations are presented.

\section{Statistical analysis}

All quantitative data were described as the mean $\pm S D$. Statistical analyses were performed using GraphPad Prism 7.0 (GraphPad Software, Inc., USA). Unpaired $t$ tests were used to compare 2 groups when appropriate. In the case of multiple groups, oneway ANOVA was employed with Tukey's post-test. $P<0.05$ was considered significant.

\section{Results}

\section{Characteristics of nor-exo and hyp-exo}

To examine the characteristics of nor-exo and hyp-exo, the exosomes were recovered from ECFC conditioned media (ECFCCM), followed by TEM, NTA, and Western blot analysis. TEM of ECFC-derived exosomes revealed the presence of spherical structures with a typical size of $30-150 \mathrm{~nm}$ in diameter (Figure 1A). Furthermore, the expression of the exosome markers Alix, CD9, and CD63 was confirmed by Western blot analysis (Figure 1B). To test exosome purity, calnexin, which has been proposed as a negative marker of exosomes, was also detected. To further investigate the size-distribution profiles of ECFC-derived exosomes, we performed size detection using NTA, which revealed size peaks of $110 \mathrm{~nm}$ (nor-exo group) and $114 \mathrm{~nm}$ (hyp-exo group), respectively (Figure 1C). Size peak particles concentrations in the nor-exo group were higher than in the hyp-exo group $\left(5.49 \times 10^{6}\right.$ particles $/ \mathrm{ml}$ vs. $3.33 \times 10^{6}$ particles $/ \mathrm{ml}$ ). These results indicated that we had obtained typical exosomes from ECFC-CM in both the normoxia and hypoxia 


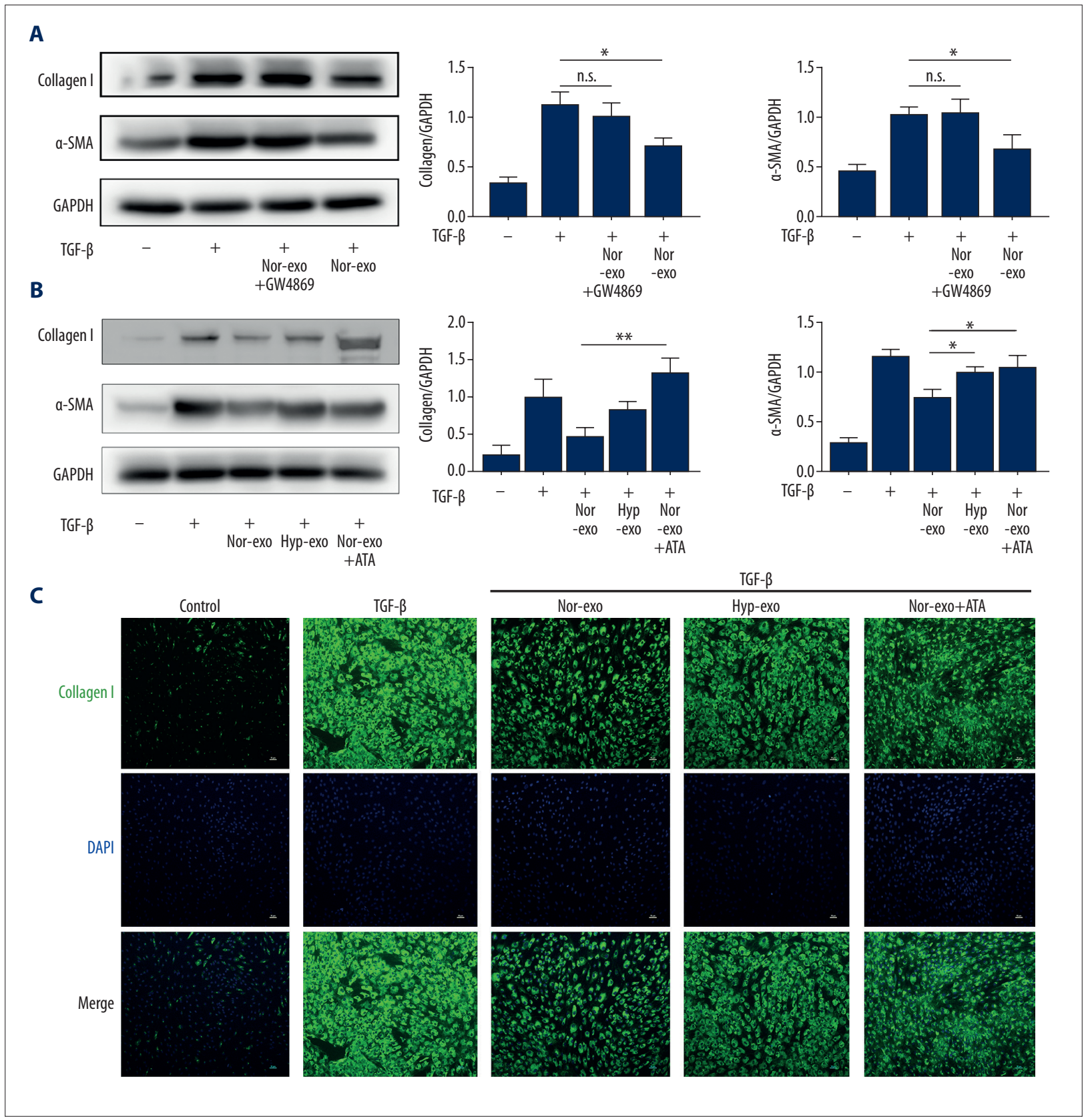

Figure 2. In vitro effects of nor-exo and hyp-exo on Col-1 $\alpha$ and $\alpha$-SMA expression in cardiac fibroblasts. Cardiac fibroblasts were incubated with nor-exo $(75 \mu \mathrm{g} / \mathrm{ml})$ or GW4869-treated nor-exo $(75 \mathrm{mg} / \mathrm{ml})$ and then stimulated with TGF- $\beta_{1}(15 \mathrm{ng} / \mathrm{ml})$ for 48 h (A). In part B, the anti-fibrotic effects of hyp-exo $(75 \mathrm{mg} / \mathrm{ml})$ and nor-exo $(75 \mu \mathrm{g} / \mathrm{ml})$ in combination with ATA $(25 \mu \mathrm{M})$ were also detected. Col-1 $\alpha$ was observed in cardiac fibroblasts treated with different exosomes using a fluorescence microscope (C). Mean $\pm \mathrm{SD}, \mathrm{n}=3$, ANOVA followed by Tukey's post-test. ${ }^{*} P<0.05 ;{ }^{* *} P<0.01$.

treatment groups. There were no significant differences between the 2 groups of exosomes with regard to their characteristics, except for particles concentration.

\section{Hypoxia attenuated the anti-fibrotic effects of ECFC- derived exosomes in vitro}

Hypoxia reportedly impairs the repair capacity of ECFCs [14,15], although its effects on exosome functions remain unknown. Therefore, we assessed the different therapeutic potencies of nor-exo and hyp-exo. 


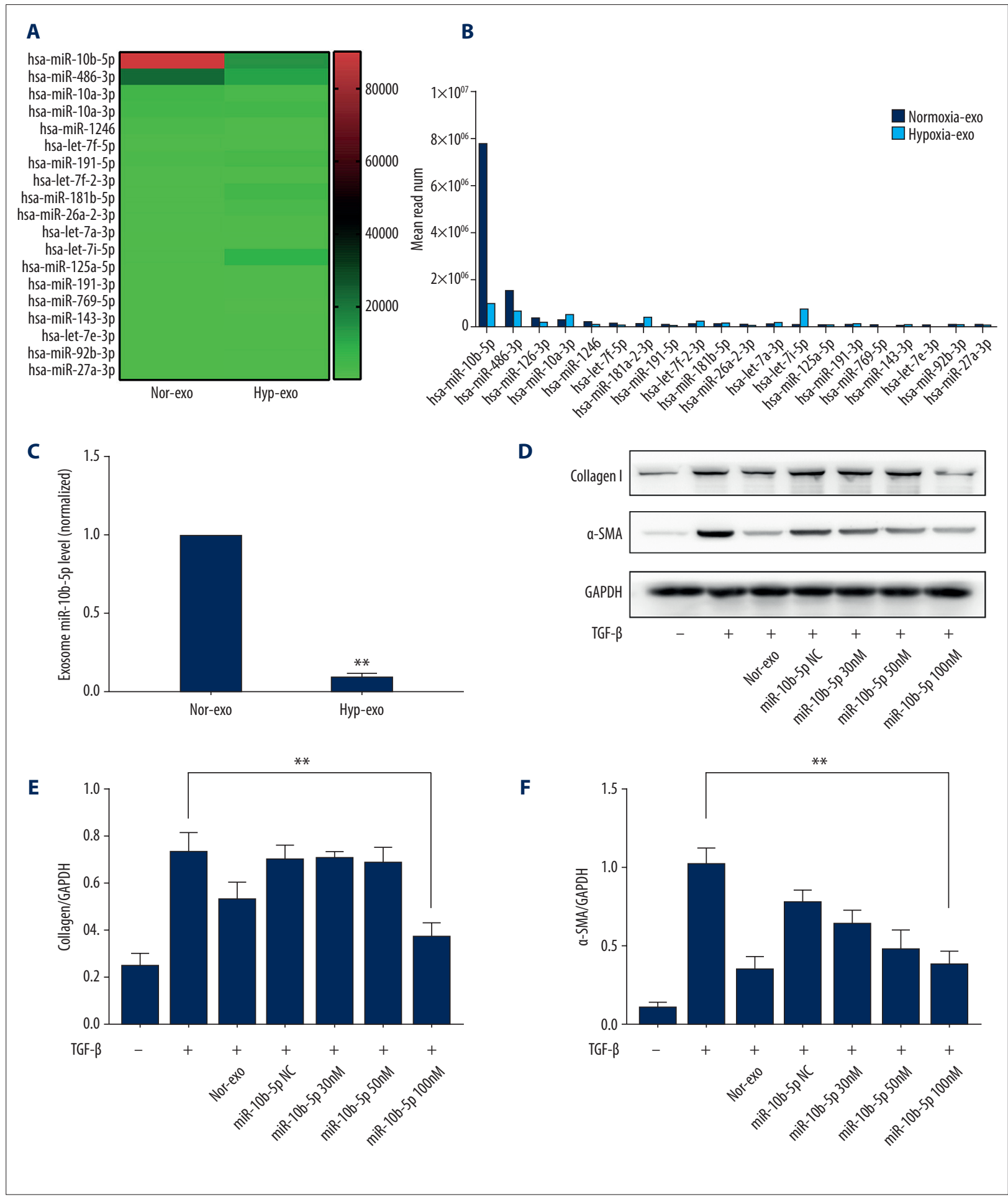

Figure 3. Characterization of miRNAs in ECFC-derived exosomes and the role of miR-10b-5p in protecting fibroblasts from activation. Next-generation RNA sequencing results (A, B). RT-qPCR results of the most highly differential miRNAs between nor-exo and hyp-exo (C). Effects of nor-exo and miR-10b-5p on Col- $1 \alpha$ and $\alpha$-SMA expression (D, E, F). Mean \pm SD, $n=3$, by $t$ test (C), ANOVA followed by Tukey's post-test (E, F). ${ }^{*} P<0.05 ;{ }^{* *} P<0.01$. 
To investigate the potential effects of exosomes on fibrosis activation, we first treated cardiac fibroblasts with nor-exo before stimulation with TGF- $\beta$. TGF- $\beta$ stimulation significantly increased Col- $1 \alpha$ and $\alpha$-SMA expression levels, which were significantly attenuated by nor-exo (Figure 2A, Supplementary Figure 3). To validate the role of exosomes, we treated ECFCs with a neutral sphingomyelinase 2 (N-SMase2) inhibitor, GW4869, which inhibited exosome secretion [27], followed by a sequential centrifugation process. Next, the centrifugation products were added to fibroblasts, and Col- $1 \alpha$ and $\alpha$-SMA expression profiles were examined. The results showed that GW4869 dramatically reversed the nor-exo-induced reduction in Col- $1 \alpha$ and $\alpha$-SMA (Figure 2A). These results clearly demonstrated that normoxiaECFC-derived exosomes, but not other components from culture medium, reduced fibroblast activation.

We further examined the anti-fibrotic effects of hyp-exo in cardiac fibroblasts in vitro. As indicated in Figure $2 \mathrm{~B}$, nor-exo significantly suppressed both $\mathrm{Col}-1 \alpha$ and $\alpha$-SMA expression, while the effects of hyp-exo were similar to TGF- $\beta$ alone (Figure 2B). However, it remained unclear whether the attenuated effects of hyp-exo were altered by the change of exosomes-carried miRNAs (miRs), although such a relationship has been documented in a wide range of studies $[7,27,28]$. Therefore, we incubated nor-exo combined with aurintricarboxylic acid (ATA), an RNA-induced silencing complex inhibitor that has been used to suppress the effects of miRs [29]. As shown in Figure 2B, ATA nearly completely abolished the therapeutic effects conferred by nor-exo. Immunofluorescence cytochemistry results were consistent with the observed expression (Figure 2C). Taken together, these data show that exosomes carrying miRs play a protective role in the reduction of cardiac fibroblast activation.

\section{Characterization of miRNAs in ECFC-derived exosomes and the role of miR-10b-5p in protecting fibroblasts from activation}

Our results indicated that miRNAs play a role in the anti-fibrotic effects of exosomes, but the underlying mechanism of miR function is unknown. Therefore, we used next-generation sequencing to identify relevant miRs. A total of 721 miRs were upregulated, and 1140 miRs were downregulated in hyp-exo (see Supplementary Figure 4). Based on the read number of each miRNA in nor-exo, which represents the abundance of miRNA expression, we selected the top 20 miRNAs for further investigation. Figure $3 \mathrm{~A}$ shows the relative changes in these miRNAs in hyp-exo compared with those in nor-exo. Among these miRs, miR-10b-5p showed the highest expression in normoxia exosomes, as indicated by its relatively large read number, which was greater than the sum of the other top 19 miRNAs. However, its expression was dramatically decreased in the hypoxia group (Figure 3B), which was confirmed by subsequent RT-PCR results (Figure $3 C$ ).
Next, we examined the role of miR-10b-5p in fibroblast activation. As expected, these results showed that when the miR$10 \mathrm{~b}-5 \mathrm{p}$ concentration reached $100 \mathrm{nM}$, the reduction in Col$1 \alpha$ and $\alpha$-SMA expression was similar to that in the normoxia exosome group (Figure 3D-3F). Taken together, these results showed that miR-10b-5p induced anti-fibrotic effects.

\section{Targeting of HDAC4 and Smurf1 mRNAs by miR-10b-5p}

Considering that miRNAs are responsible for the differing effects of hyp-exo and nor-exo on fibroblast activation, and TGF- $\beta$ is one of the major classical factors inducing fibroblast activation, we next sought to identify whether miR-10b-5p affects TGF- $\beta$-associated fibroblast activation. The RNAhybrid, miRan$\mathrm{da}$, and TargetScan databases were used to predict targets of miR-10b-5p, and 100 potential targets are shown (Figure 4A). Additionally, 255 genes involved in the TGF- $\beta$ signaling pathway were identified from the KEGG database, and 4 genes were found in both datasets (SMURF1, mRNA ID: NM_001199847; NBL1, mRNA ID: NM_001204086; ID2, mRNA ID: NM_002166; HDAC4, mRNA ID: NM_006037; Figure 4A, 4B).

To validate the results of the bioinformatics predictions, a dual-luciferase reporter gene assay was performed. A miR-10b$5 p$ mimic significantly reduced the relative luciferase activity of the Smurf1-3'-UTR and HDAC4-3'-UTR vectors (Figure 4C). However, luciferase activity from the ID2-3'-UTR and NBL2-3'UTR vectors remained unchanged in the presence of miR-10b$5 p$. Taken together, these results confirmed that Smurf1 and HDAC4 mRNAs are direct targets of miR-10b-5p.

We also aimed to understand whether miR-10b-5p downregulated the expression of HDAC4 and Smurf1, similar to our observations with nor-exo (Figure 4D-4F). Our results indicated that $\mathrm{miR}-10 \mathrm{~b}-5 \mathrm{p}$ regulated the TGF- $\beta$ signaling pathway via the decreased expression of HDAC4 and Smurf1.

\section{Expression of $\mathrm{N}-\mathrm{SM} M a s e 2$ was consistent with changes in miR-10b-5p in hyp-exo}

When we compared miR-10b-5p levels between normoxiatreated ECFCs and hypoxia-treated ECFCs, no difference was observed, which was inconsistent with the change in miR-10b$5 p$ in hyp-exo (Figure 5A). Furthermore, N-SMase 2 is well accepted to play an important role in exosome biogenesis [30,31]. Thus, we examined $\mathrm{N}$-SMase 2 expression during hypoxia. The results showed that $\mathrm{N}$-SMase 2 expression was decreased in hypoxia-treated ECFCs (Figure 5B). The results indicated an impairment of miR-10b-5p packaging in ECFCs during hypoxia. In addition, N-SMase 2 mRNA levels in ECFCs significantly increased during hypoxia, suggesting that hypoxia reduces $\mathrm{N}$-SMase post-transcriptionally (Figure 5C). 


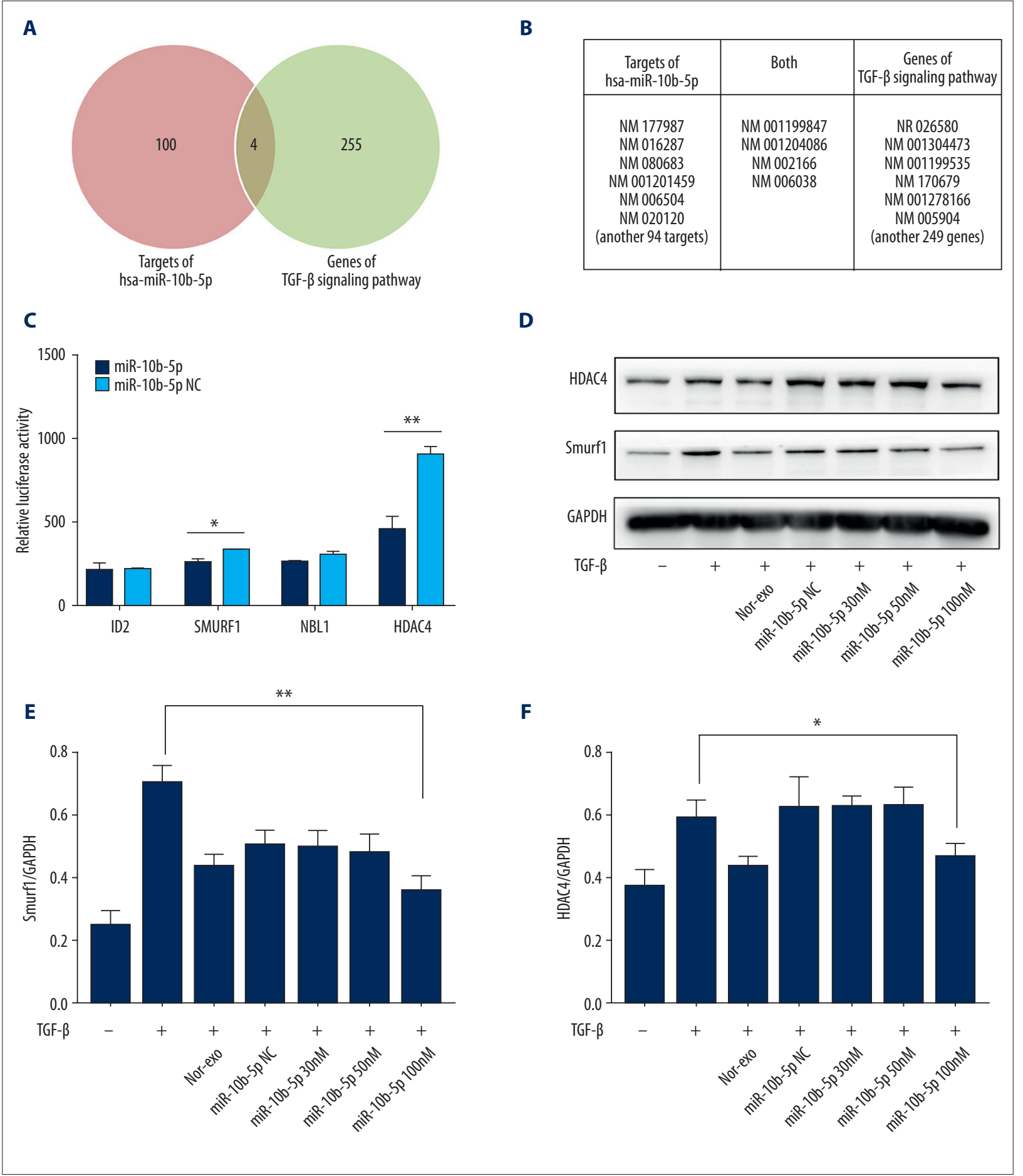

Figure 4. Targeting of HDAC4 and Smurf1 mRNAs by miR-10b-5p. Predicted mRNA targets of miR-10b-5p that overlapped with the TGF- $\beta$ signaling pathway (A, B). Validation of mRNA targets of miR-10b-5p using a dual-luciferase reporter gene assay (C). Effects of nor-exo and miR-10b-5p on HDAC4 and Smurf1 expression (D-F). Mean $\pm S D, n=3$, ANOVA followed by Tukey's posttest. * $P<0.05 ;{ }^{* *} P<0.01$. 

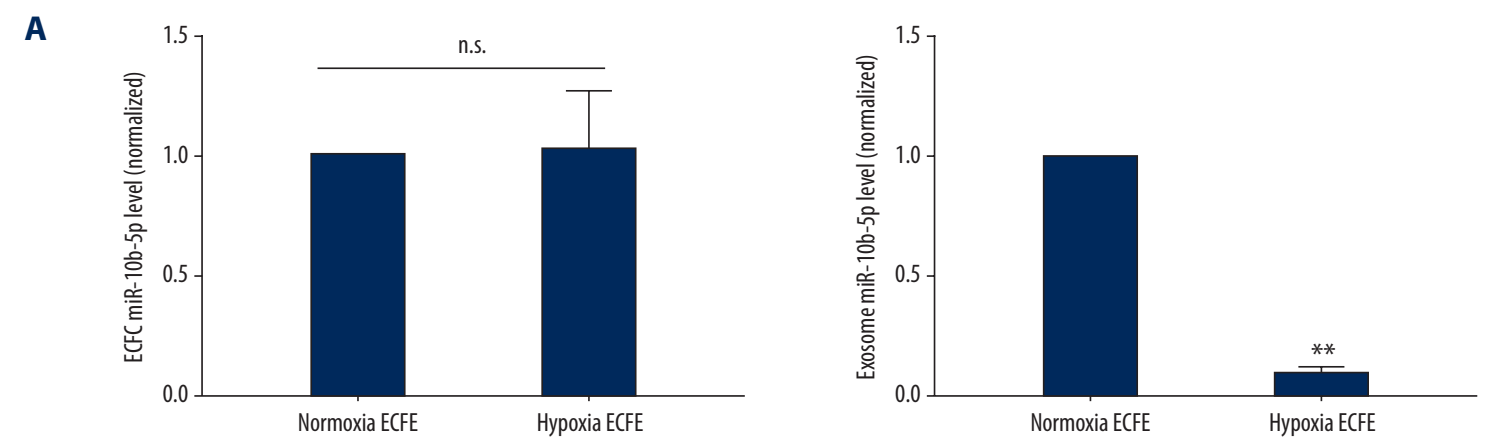

B
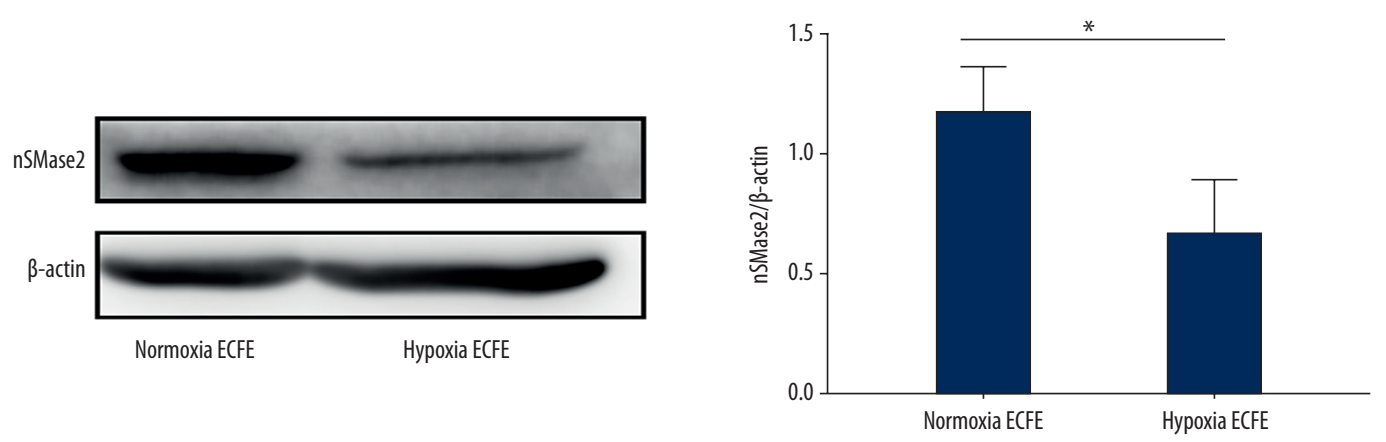

C

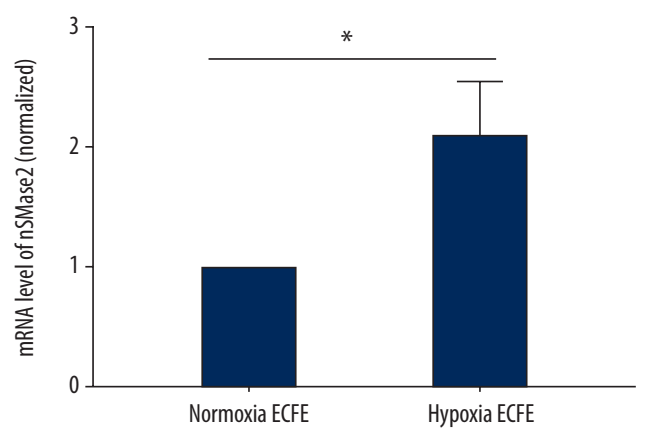

Figure 5. Expression of $\mathrm{N}-\mathrm{S}$ mase 2 in hypoxia-treated ECFCs. ECFCs were incubated in a hypoxia chamber for $72 \mathrm{~h}$, and miR-10b-5p levels in cells and hyp-exo were examined using PCR (A). N-SMase2 expression of normoxic and hypoxic ECFCs was detected by WB (B), and mRNA levels were also determined (C). Mean $\pm S D, n=3$, by $t$ test $(A, B)$, ANOVA followed by Tukey's posttest $(\mathbf{C}) .{ }^{*} P<0.05 ;{ }^{* *} P<0.01$

\section{Discussion}

ECFCs may be a promising stem cell therapy for treating ischemic diseases, particularly MI. However, the micro-environment around infarcted zones is deleterious to ECFC growth and proper function. Exosomes have emerged as a promising therapeutic alternative, and the effects of hypoxia on ECFCderived exosomes are currently not conclusive. Our study confirmed that the anti-fibrotic effects of exosomes from ECFCs are ameliorated under hypoxic conditions. Moreover, using high-throughput methods, we found that decreased levels of miR-10b-5p, which targets HDAC4 and SMURF1 mRNA, may account for the attenuated therapeutic potency of hyp-exo. Taken together, the results of our study may deepen our understanding of the reparative limitations of ECFCs after MI.
Hypoxia-impaired ECFC function has been described. For example, several studies have reported decreased ECFC proliferation, migration, angiogenesis, and vessel formation under hypoxic conditions [12-14]. However, to the best of our knowledge, this is the first study to directly demonstrate that exosomes from ECFCs ameliorate cardiac fibrosis. Indeed, although previous studies have reported that microvesicles from ECFCs protect cardiomyocytes from hypertrophy and apoptosis, and exosomes from these cell reduce ischemic kidney injury [7,32], no study has directly assessed the effects of exosomes or microvesicles on the ischemic heart. However, the injection of ECFC-derived exosomes has been shown to be safe, and thus, our study may provide the first description of a feasible clinical therapeutic treatment. 


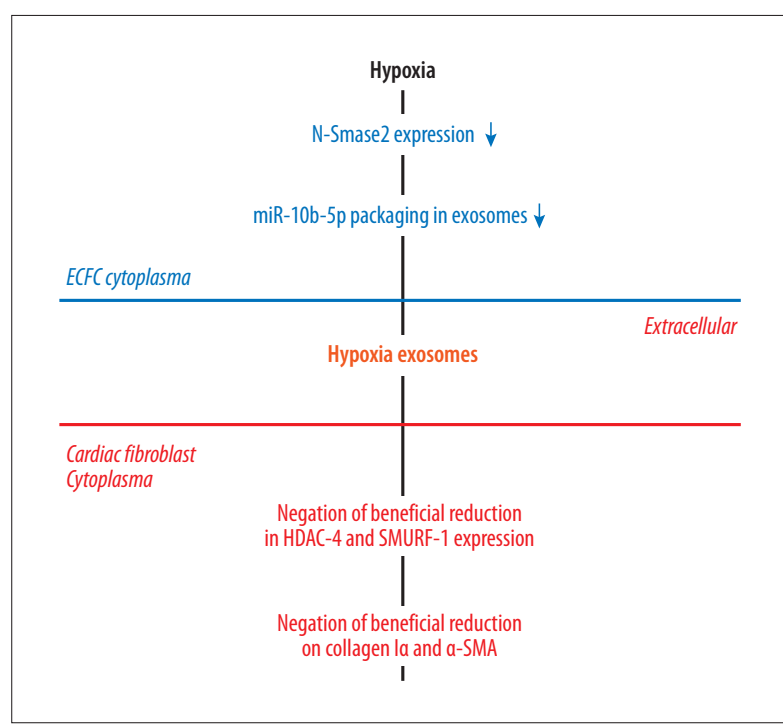

Figure 6. General overview of the hypoxia and ECFC-derived exosomes. Hypoxia decreases $\mathrm{N}-\mathrm{Smase} 2$ expression in ECFCs, which results in down-regulation of miR$10 \mathrm{~b}-5 \mathrm{p}$ packing in exosomes. As a result, negation of beneficial reduction in HDAC4 and SMURF1 expression and negation of beneficial reduction on Col and SMA in Cardiac Fibroblasts are happened.

The mechanism underlying how exosomes exert their protective effects is also important. Classical effects of ECFCs, such as the promotion of angiogenesis, have been validated. Consistent with such a finding, angiogenic effects of ECFCderived exosomes have also been reported. Li et al. found that ECFC-derived exosomes accelerated re-endothelialization [33]. Similarly, a recent study showed that exosomes from ECFCs induced mesenchymal-endothelial transition and promoted angiogenesis via a reduction in high-mobility group box-1 protein B1 expression [34]. In addition to angiogenesis, ECFC-derived exosomes also reduced renal cell apoptosis in ischemic kidney injury [17]. Our study showed that ECFC-derived exosomes antagonized fibrosis by inhibiting cardiac fibroblast activation and the secretion of extracellular matrix. The non-angiogenesis effects of ECFC-derived exosomes described in the present study widen the known mechanisms of ECFC exosomes and provide additional clues for subsequent studies in this field.

Most importantly, we revealed a mechanism underlying decreased therapeutic potency of hyp-exo: upon hypoxia, alterations in exosome cargo are responsible for the decreased protective effects of ECFC-derived exosomes, manifesting as reduced fibroblast activation. miRNAs carried by exosomes are the most well-studied, but the differences between nor-exo and hyp-exo have remained uncharacterized. In addition, it is unclear whether alterations to miRNAs explain the distinct effects of the 2 exosome populations. We used RNA sequencing to compare all the miRNA expression profiles and found that miR-10b-5p was abundantly expressed in both ECFCs and their exosome. miR$10 b-5 p$ expression was also downregulated to the greatest extent in hyp-exo, which might contribute to the potential mechanism underlying the reduced therapeutic effects observed with these exosomes. Although no study has directly demonstrated an association between miR-10b-5p and fibrosis, both human and animal studies investigating cardiac fibrosis with different etiologies have consistently shown that miR-10b-5p is inversely correlated with fibrosis $[35,36]$. Moreover, the Smad/HDAC pathway, which is the classical and primary mechanism leading to fibrosis, has been confirmed as a target of miR-10b-5p. Thus, our study suggests that decreased miR-10b-5p may be responsible for the attenuated therapeutic effects of hyp-exo (Figure 6).

Despite our findings, some limitations of the present study should be acknowledged. First, although we demonstrated that hyp-exo is less effective at reducing fibrosis, we did not provide direct evidence to demonstrate alterations to miR$10 b-5 p$ in ECFC-derived exosomes in the infarcted heart model; therefore, there is a lack of evidence directly showing the role of miR-10b-5p. In addition, although our data indicated that a change in N-SMase2 might be involved in the alterations to miR-10b-5p in hypoxia-derived exosomes, we did not determine how hypoxia affects the expression of N-SMase 2 or how N-SMase 2 affects the packaging of miR-10b-5p in exosomes. Thus, additional studies to explore how hypoxia affects the packaging of miR-10b-5p in exosomes will be meaningful.

\section{Conclusions}

In summary, despite these limitations, the present study demonstrated that human ECFC-derived exosomes reduce cardiac fibroblast activation, which was greatly attenuated when these exosomes were exposed to hypoxia. RNA sequencing revealed that miR-10-5p was abundantly enriched in ECFC-derived exosomes and was dramatically decreased in hypoxia-cultured ECFCderived exosomes. Moreover, bioinformatics analyses and dualluciferase reporter gene assays demonstrated that HDAC4 and Smurf1, which play important roles in the TGF- $\beta$ signaling pathway, are targets of miR-10b-5p. Taken together, these results suggest that the use of miR-10b-5p-enriched exosomes may be a viable strategy for reducing fibroblast activation after MI.

\section{Conflict of interests}

None.

\section{Availability of data and material}

The datasets used during the current study and all supplemental materials are available from the corresponding author on reasonable request. 


\section{Supplementary Figures}

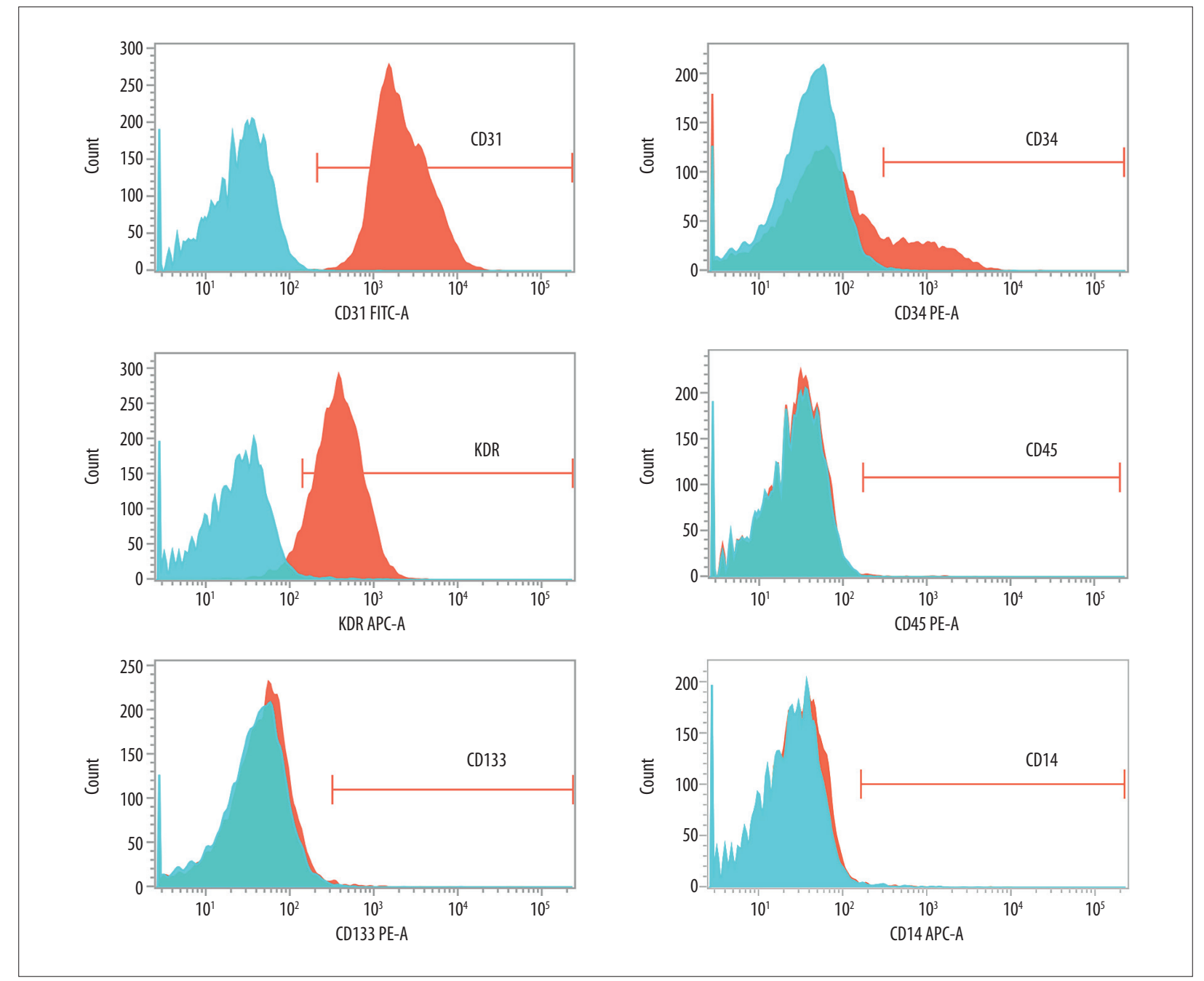

Supplementary Figure 1. ECFCs characterization. ECFCs immunolabeled with a control antibody are presented with blue line and specific antibody are presented with red line.

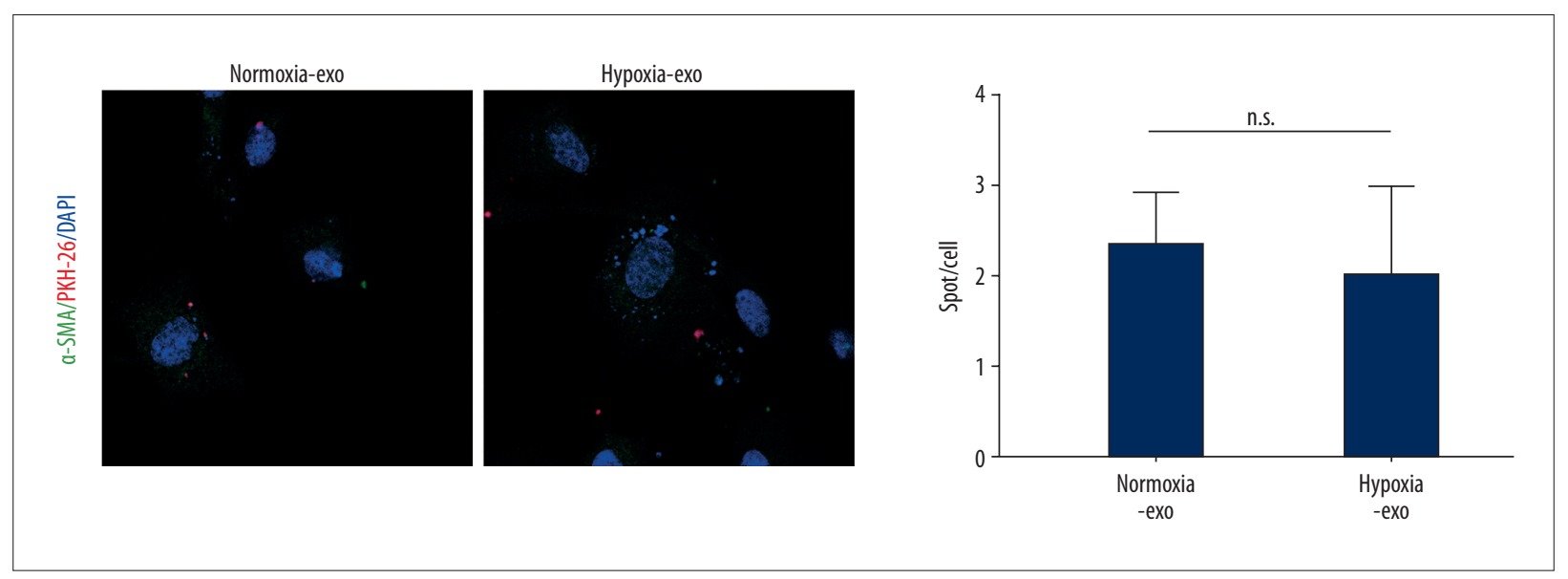

Supplementary Figure 2. Cellular uptake of exosomes. Mean $\pm S D, n=3$, by $t$ test.

This work is licensed under Creative Common AttributionNonCommercial-NoDerivatives 4.0 International (CC BY-NC-ND 4.0)

\section{7}

Indexed in: [Current Contents/Clinical Medicine] [SCI Expanded] [ISI Alerting System] [ISI Journals Master List] [Index Medicus/MEDLINE] [EMBASE/Excerpta Medica] [Chour Jaster List] [ndex Medicus/MEDLNE] [EMBASE/Excerpta Medica] [Chemical Abstracts/CAS] 


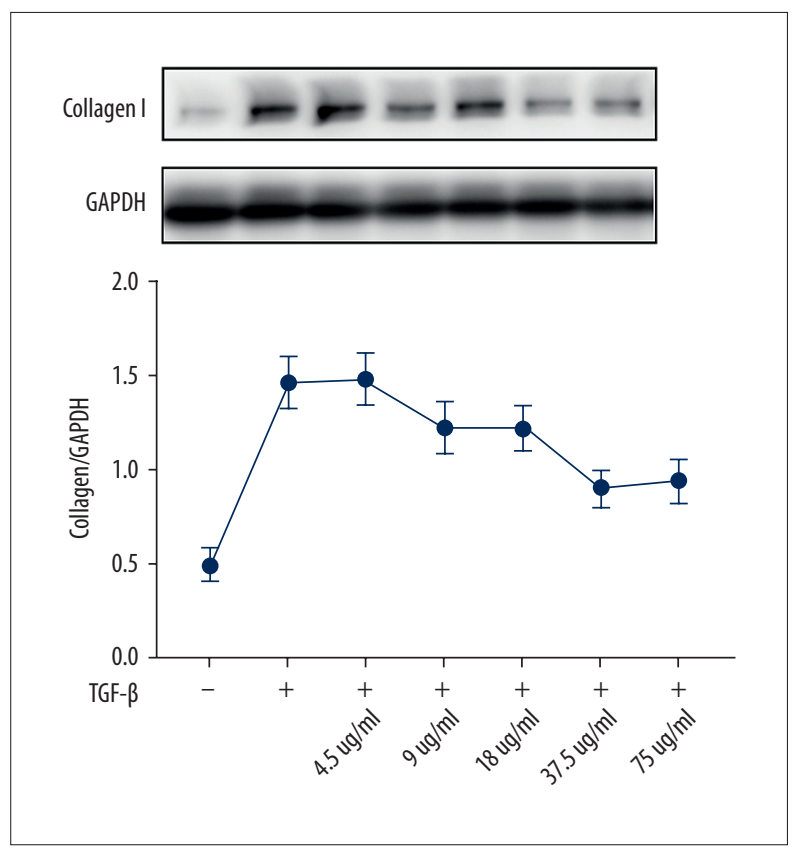

Supplementary Figure 3. Expression of collagen I was reduced by nor-exo in a dose-dependent manner.

\section{References:}

1. Benjamin EJ, Blaha MJ, Chiuve SE et al. Heart disease and stroke statistics - 2017 update: A report from the American Heart Association. Circulation, 2017; 135: e146-e603

2. Authors/Task Force Members, Windecker S, Kolh P, Alfonso F et al: 2014 ESC/EACTS Guidelines on myocardial revascularization: The Task Force on Myocardial Revascularization of the European Society of Cardiology (ESC) and the European Association for Cardio-Thoracic Surgery (EACTS)Developed with the special contribution of the European Association of Percutaneous Cardiovascular Interventions (EAPCI). Eur Heart J, 2014; 35: 2541-619

3. Leistner DM, Fischer-Rasokat U, Honold J et al: Transplantation of progenitor cells and regeneration enhancement in acute myocardial infarction (TOPCARE-AMI): Final 5-year results suggest long-term safety and efficacy. Clin Res Cardiol, 2011; 100: 925-34

4. Quyyumi AA, Waller EK, Murrow J et al: CD34(+) cell infusion after ST elevation myocardial infarction is associated with improved perfusion and is dose dependent. Am Heart J, 2011; 161: 98-105

5. Kim SW, Jin HL, Kang SM et al: Therapeutic effects of late outgrowth endothelial progenitor cells or mesenchymal stem cells derived from human umbilical cord blood on infarct repair. Int J Cardiol, 2016; 203: 498-507

6. Kazakov A, Muller P, Jagoda P et al: Endothelial nitric oxide synthase of the bone marrow regulates myocardial hypertrophy, fibrosis, and angiogenesis. Cardiovasc Res, 2012; 93: 397-405

7. Vinas JL, Burger D, Zimpelmann J et al: Transfer of microRNA-486-5p from human endothelial colony forming cell-derived exosomes reduces ischemic kidney injury. Kidney Int, 2016; 90: 1238-50

8. Whiteside TL: Exosomes and tumor-mediated immune suppression. J Clin Invest, 2016; 126: 1216-23

9. Zhang HG, Grizzle WE: Exosomes: A novel pathway of local and distant intercellular communication that facilitates the growth and metastasis of neoplastic lesions. Am J Pathol, 2014; 184: 28-41

10. Liao SY, Tse HF: Multipotent (adult) and pluripotent stem cells for heart regeneration: What are the pros and cons? Stem Cell Res Ther, 2013; 4: 151

11. Radosinska J, Bartekova M: Therapeutic potential of hematopoietic stem cell-derived exosomes in cardiovascular disease. Adv Exp Med Biol, 2017; 998: 221-35

12. Kim J, Shapiro L, Flynn A: The clinical application of mesenchymal stem cells and cardiac stem cells as a therapy for cardiovascular disease. Pharmacol Ther, 2015; 151: 8-15

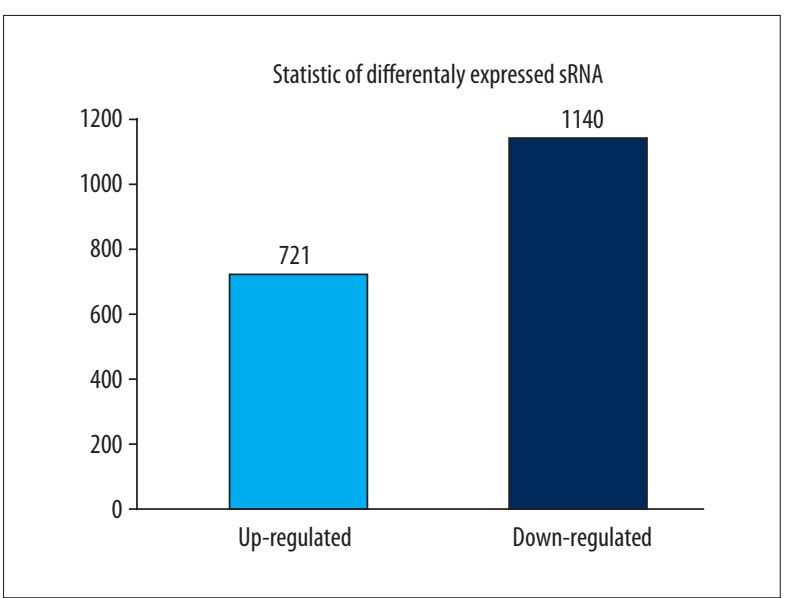

Supplementary Figure 4. Characterization of ECFC-derived exosomes. Change of miRNAs in hyp-exo when compared with nor-exo.

13. Cheng K, Li TS, Malliaras K et al: Magnetic targeting enhances engraftment and functional benefit of iron-labeled cardiosphere-derived cells in myocardial infarction. Circ Res, 2010; 106: 1570-81

14. Hookham MB, Ali IH, O'Neill CL et al: Hypoxia-induced responses by endothelial colony-forming cells are modulated by placental growth factor. Stem Cell Res Ther, 2016; 7: 173

15. He M, Ma S, Cai $Q$ et al: Hypoxia induces the dysfunction of human endothelial colony-forming cells via HIF-1alpha signaling. Respir Physiol Neurobiol, 2017; 247: 87-95

16. Dincer UD: Fetal exposure to a diabetic intrauterine environment resulted in a failure of cord blood endothelial progenitor cell adaptation against chronic hypoxia. Stem Cells Cloning, 2015; 8: 1-14

17. Burger D, Vinas JL, Akbari S et al: Human endothelial colony-forming cells protect against acute kidney injury: Role of exosomes. Am J Pathol, 2015; 185: 2309-23

18. Mai J, Qiu Q, Lin YQ et al: Angiotensin II-derived reactive oxygen species promote angiogenesis in human late endothelial progenitor cells through heme oxygenase-1 via ERK1/2 and AKT/PI3K pathways. Inflammation, 2014; 37: 858-70

19. Smit NP, Westerhof W, Menko WJ et al: Stimulation of cultured melanocytes in medium containing a serum substitute: Ultroser-G. Pigment Cell Res, 1995; 8: 19-27

20. Wunderlich K, Knorr M, Dartsch PC: Serum-free cultivation of bovine lens epithelial cells. Graefes Arch Clin Exp Ophthalmol, 1994; 232: 355-60

21. Epple LM, Griffiths SG, Dechkovskaia AM et al: Medulloblastoma exosome proteomics yield functional roles for extracellular vesicles. PLoS One, 2012 7: e42064

22. Thery C, Amigorena S, Raposo G, Clayton A: Isolation and characterization of exosomes from cell culture supernatants and biological fluids. Curr Protoc Cell Biol, 2006; Chapter 3: Unit 322

23. Xu R, Greening DW, Zhu $\mathrm{H}-\mathrm{J}$ et al: Extracellular vesicle isolation and characterization: Toward clinical application. J Clin Invest, 2016; 126: 1152-62

24. Gao W, Liu H, Yuan J et al: Exosomes derived from mature dendritic cells increase endothelial inflammation and atherosclerosis via membrane TNFalpha mediated NF-kappaB pathway. J Cell Mol Med, 2016; 20: 2318-27 
25. Zhang HF, Wu MX, Lin YQ et al: IL-33 promotes IL-10 production in macrophages: A role for IL-33 in macrophage foam cell formation. Exp Mol Med, 2017; 49: e388

26. Zhou X, Jiao Z, Ji J et al: Characterization of mouse serum exosomal small RNA content: The origins and their roles in modulating inflammatory response. Oncotarget, 2017; 8: 42712-27

27. Bang C, Batkai S, Dangwal S et al: Cardiac fibroblast-derived microRNA passenger strand-enriched exosomes mediate cardiomyocyte hypertrophy. J Clin Invest, 2014; 124: 2136-46

28. Barile L, Lionetti V, Cervio E et al: Extracellular vesicles from human cardiac progenitor cells inhibit cardiomyocyte apoptosis and improve cardiac function after myocardial infarction. Cardiovasc Res, 2014; 103: 530-41

29. Tan GS, Chiu CH, Garchow BG et al: Small molecule inhibition of RISC loading. ACS Chem Biol, 2012; 7: 403-10

30. Shamseddine AA, Airola MV, Hannun YA: Roles and regulation of neutral sphingomyelinase-2 in cellular and pathological processes. Adv Biol Regul, 2015; 57: 24-41
31. Kapustin AN, Chatrou MLL, Drozdov I et al: Vascular smooth muscle cell calcification is mediated by regulated exosome secretion. Circul Res, 2015 116: $1312-23$

32. Bitzer M, Ben-Dov IZ, Thum T: Microparticles and microRNAs of endothelial progenitor cells ameliorate acute kidney injury. Kidney Int, 2012; 82: 375-77

33. Li X, Chen C, Wei $L$ et al: Exosomes derived from endothelial progenitor cells attenuate vascular repair and accelerate reendothelialization by enhancing endothelial function. Cytotherapy, 2016; 18: 253-62

34. Ke X, Yang D, Liang J et al: Human endothelial progenitor cell-derived exosomes increase proliferation and angiogenesis in cardiac fibroblasts by promoting the mesenchymal-endothelial transition and reducing high mobility group box 1 protein B1 expression. DNA Cell Biol, 2017; 36: 1018-28

35. Fang L, Ellims AH, Moore XL et al: Circulating microRNAs as biomarkers for diffuse myocardial fibrosis in patients with hypertrophic cardiomyopathy. J Transl Med, 2015; 13: 314

36. Yang Q, Cui J, Wang P et al: Changes in interconnected pathways implicating microRNAs are associated with the activity of apocynin in attenuating myocardial fibrogenesis. Eur J Pharmacol, 2016; 784: 22-32 